\title{
Tensile Tests of Polypropylene Monitored by SAXS. Comparing the Stretch-Hold Technique to the Dynamic Technique
}

\author{
NORBERT STRIBECK, ${ }^{1}$ ULRICH NÖCHEL, ${ }^{1}$ SÉRGIO S. FUNARI, ${ }^{2}$ TOM SCHUBERT ${ }^{2}$ \\ ${ }^{1}$ Department of Chemistry, University of Hamburg, Institute TMC, Bundesstr. 45, D-20146 Hamburg, Germany \\ ${ }^{2}$ HASYLAB at DESY, Notkestr. 86, D-22603 Hamburg, Germany
}

Received 4 November 2007; revised 16 December 2007; accepted 17 December 2007

DOI: 10.1002 / polb.21403

Published online 4 February 2008 in Wiley InterScience (www.interscience.wiley.com).

\begin{abstract}
Advanced instrumentation at synchrotron beamlines facilitates considerable reduction of the exposure required for the recording of low-noise small-angle X-ray scattering (SAXS) patterns. Concerning the monitoring of tensile tests on polymer materials by SAXS means that change-over from the stretch-hold technique to more practical dynamic straining is possible, although the strain rate is still by a factor of 100 lower than that relevant in industry and service. As hard-elastic polypropylene (PP) is tested, considerably different scattering patterns are recorded after switching to the dynamic technique. Even though during exposure the elongation is no longer constant when applying the dynamic technique, the images collected in stretch-hold technique appear much more blurred. The observed immediate relaxation of nanostructure induced by stopping the extensometer is analyzed by means of the multidimensional chord distribution function (CDF): The extension of the crystalline lamellae is increasing. Their thicknesses are becoming non-uniform. The range of order is shortening. Cross-hatched lamellae are formed. () 2008 Wiley Periodicals, Inc. J Polym Sci Part B: Polym Phys 46: 721-726, 2008
\end{abstract}

Keywords: drawing; microstructure; poly(propylene); SAXS; tensile testing

\section{INTRODUCTION}

Studies, in which small-angle X-ray scattering (SAXS) is used to monitor the deformation of polymer materials are carried out in order to answer questions of some practical relevance: What are the mechanisms of nanostructure evolution by which the material responds to mechanical loading? What is the constitution of the polymer nanocomposite that imprints tailored properties to the material like special toughness or low

Correspondence to: N. Stribeck (E-mail: norbert.stribeck@ desy.de)

Journal of Polymer Science: Part B: Polymer Physics, Vol. 46, 721-726 (2008)

(C) 2008 Wiley Periodicals, Inc. fatigue? Such advanced materials are urgently sought after e.g. in automotive industry, in order to accomplish the goals of climate protection by reduction of weight.

Those deformation studies which are aiming at quantitative analysis of the scattering pattern are presently experiencing a conversion from the socalled stretch-hold technique ${ }^{1}$ to an in situ study of nanostructure evolution during dynamical tensile tests. The reason is the fact that with modern equipment at synchrotron radiation sources the exposure required for a low-noise SAXS pattern has dropped below $1 \mathrm{~min}$.

So far, it has been necessary to stop the extensometer at various elongations, because the recording of a low-noise SAXS pattern required 
ample time. Now it is possible to switch from the static to the dynamic technique, i.e., to expose without stopping the extensometer - provided the strain rate $\left(\dot{\varepsilon} \approx 10^{-3} \mathrm{~s}^{-1}\right)$ is kept low. Using this technique we have carried out several still unpublished studies on polymer materials, but before changing over to the dynamic technique the question must be answered, if there is a difference between the patterns obtained with the static technique and those measured with the new dynamic one.

In principle, dynamic in situ investigations of deformation by means of X-ray scattering are not new. Early in the 1990's scientists at the SRS in Daresbury, Great Britain, have relinquished the postulate of a low-noise scattering pattern using instruments ${ }^{2}$ equipped with a very fast detector. The recorded scattering patterns ${ }^{3}$ are sufficient for qualitative interpretation. In this environment, structure evolution can be studied at strain rates of $\dot{\varepsilon} \approx 10^{-1} \mathrm{~s}^{-1}$, which are common in industrial processes. A selection of the studies performed with this instrument ${ }^{4-7}$ demonstrates both its power and its limits. To our knowledge, the first article on polymer deformation based on low-noise patterns with an exposure of $15 \mathrm{~s}$ and a cycle time of $30 \mathrm{~s}$ has been published by Chen et al. ${ }^{8}$

Even though the limited dynamical option has been available during the past 2 decades, most of the working groups have decided not to sacrifice data quality for the benefit of short exposure, as can be verified from the long list of published studies from which we have picked our own and some foreign references. ${ }^{9-29}$ So one of the aims of this study is to contribute to the assessment of the peculiar value of the older studies.

\section{EXPERIMENTAL}

\section{Material}

Commercial hard-elastic ${ }^{30,31}$ polypropylene (PP) film (CelGard-PP ${ }^{\circledR}$, Lot \#884, as extruded by Hoechst-Celanese) of $25 \mu \mathrm{m}$ thickness is studied. The films are produced $^{32}$ from the isotactic PP grade Hercules Profax 6301 with a melt index $15.0\left(230{ }^{\circ} \mathrm{C}\right)$ ASTM-D-1238. The weight-average molecular mass of the grade is $\bar{M}_{\mathrm{w}}=128,000 \mathrm{~g} / \mathrm{mol}$. The polydispersity of the material is $\bar{M}_{\mathrm{w}} / \bar{M}_{\mathrm{n}}=4$. Test bars conforming to DIN 53504 are punched from the double-layer film resulting in samples of $50 \mu \mathrm{m}$ thickness. For this purpose, a toggle lever press manufactured by Zwick GmbH, Ulm, Germany is used. The axis of the test bar is oriented parallel to the principal axis of the uniaxially oriented films. Fiducial marks are applied by means of a common rubber stamp with a line grid of $2 \mathrm{~mm}$ distance.

\section{Laboratory Tensile Tests}

Cross-section change of the central part of the test bar during mechanical tests is studied before the synchrotron experiments in the laboratory by means of a commercial extensometer (Zwicki Z1.0/TH1S, Zwick GmbH, Ulm, Germany).

\section{SAXS Measurements}

SAXS is performed in the synchrotron beamline A2 at HASYLAB, Hamburg, Germany. The wavelength of the X-ray beam is $0.15 \mathrm{~nm}$, and the sample-detector distance is $3045 \mathrm{~mm}$. Scattering patterns are collected by a two-dimensional position sensitive marccd 165 detector (mar research, Norderstedt, Germany) operated in $2048 \times 2048$ pixel mode (79 $\mu \mathrm{m}$ quadratic pixel size) with the multiread option enabled.

\section{Tensile Test at the Beamline}

Tensile testing at the beamline is performed by means of a new self-made extensometer fit for application at HASYLAB beamlines, which has been built by one of us (T.S.). The machine performs symmetric drawing. Signals from load cell and transducer are recorded during the experiment. The sample is monitored by a TV-camera and video frames are grabbed and stored with the experimental data. The experiment control software permits to define eight intervals of different crosshead movement.

The extensometer is operated with a crosshead speed of $1 \mathrm{~mm} / \mathrm{min}$. The true elongation $\varepsilon=(\ell-$ $\left.\ell_{0}\right) / \ell_{0}$ is computed from the initial distance, $\ell_{0}$, of the two fiducial marks enclosing the beam as measured on the TV-screen and the respective actual distance, $\ell$. During dynamic tensile testing up to $\varepsilon=0.6$ the true strain rate, $\dot{\varepsilon}$, has been found to increase monotonously from $0.8 \times 10^{-3} \mathrm{~s}^{-1}$ to $1.1 \times 10^{-3} \mathrm{~s}^{-1}$.

During the genuine dynamic tensile tests, scattering patterns are recorded every $60 \mathrm{~s}$ with an exposure of $49 \mathrm{~s}$. The static stretch-hold experiment is carried out in the same way as has been done by us in previous studies. The crossheads are moved by a defined distance of usually $2 \mathrm{~mm}$. 
Directly after reaching the stop position scattering patterns are exposed for 2 min. Thereafter the next movement is performed.

\section{SAXS Data Evaluation}

The evaluation of SAXS patterns is only possible, because we have recorded low-noise images. Our analysis is resulting in the multidimensional chord distribution function (CDF). The corresponding method has been developed in $2001{ }^{33}$ Its application has repeatedly been described in detail in many of our earlier articles ( $\mathrm{cf}^{20-22,34-38}$ ). Moreover, it is exemplified in a textbook (Stribeck, ${ }^{39}$ Sect. 8.5.5). For a schematic sketch of the steps of data analysis and the extraction of structural parameters from the CDF see for example Figure 2 in Stribeck et al. ${ }^{40}$ The method is extracting the topological information on nanostructure (i.e. a two-phase topology, $\rho(\mathbf{r}) \in\left[\rho_{\text {cryst }}, \rho_{\text {amorph }}\right]$, of phases with distinct densities) from twodimensional (2D) SAXS patterns with uniaxial symmetry. The result is an "edge-enhanced autocorrelation function," $z(\mathbf{r})$, - the autocorrelation of the gradient field, $\nabla \rho(\mathbf{r})$. Thus, as a function of ghost displacement, $\mathbf{r}$, the multidimensional CDF $z(\mathbf{r})$ shows peaks wherever there are domain surface contacts between domains in $\rho(\mathbf{r})$ and in its displaced ghost. The CDF with fiber symmetry in real space, $z\left(r_{12}, r_{3}\right)$, is computed from the fiber-symmetrical SAXS pattern, $I\left(s_{12}, s_{3}\right)$, of multiphase materials. ${ }^{33} \mathbf{s}=\left(s_{12}, s_{3}\right)$ is the scattering vector with its modulus defined by $|\mathbf{s}|=s=(2 / \lambda) \sin \theta$. Here $\lambda$ is the wavelength of radiation, and $2 \theta$ is the scattering angle. In the historical context, the CDF is an extension to Ruland's interface distribution function (IDF) ${ }^{41}$ to the multidimensional case or, in a different view, the Laplacian of Vonk's multidimensional correlation function. ${ }^{42}$

\section{RESULTS AND DISCUSSION}

\section{Direct Observations in the Recorded Patterns}

Figure 1 shows the central part of selected pairs of scattering patterns, $I\left(s_{12}, s_{3}\right)$, in a pseudocolor representation (linear intensity scale). On the left side the scattering of the original material is indicated by the label $\varepsilon=0$.

Further to the right, the top row shows the data recorded during the dynamic experiment. These patterns are labeled by the average elongation, $\varepsilon$, which has been reached in the middle

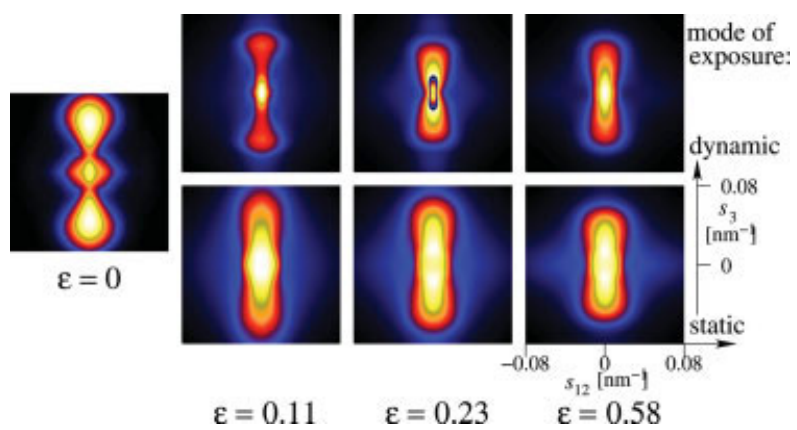

Figure 1. Comparison between SAXS patterns recorded during a dynamic (top row, $\dot{\varepsilon} \approx 10^{-3} \mathrm{~s}^{-1}$ ) tensile test of hard-elastic PP films and results from the corresponding experiment in stretch-hold technique (bottom row). Straining direction and meridian is vertical $\left(s_{3}\right)$. Equator $\left(s_{12}\right)$ is horizontal.

of the exposure interval. From the beginning to the end of the exposure interval the true elongation increases by $\Delta \varepsilon=0.037$ for the first and by $\Delta \varepsilon=0.053$ for the last presented elongation.

In the bottom row the data from the comparison experiment are presented, in which the material is subjected to the stretch-hold technique with a static measurement of the scattering pattern at constant elongation.

The major effect is just the contrary of what has been expected. Because during the recording of a pattern in the dynamic experiment the elongation has increased by $\sim 5 \%$, whereas in the static experiment the elongation has been kept constant, we have expected more blur in the patterns from the top row. Instead, the patterns in the bottom row look much more blurred. A second exposure in the hold state has returned the same images. From these observations, we conclude that immediately as the extensometer is stopped, a rapid "nanostructure relaxation" with a considerable loss of long range order takes place. Despite this general loss of long range order, the relative strength of the equatorial streak is much stronger in the stretch-hold patterns than in the dynamic patterns.

Thus this comparative experiment demonstrates that stopping the extensometer may induce considerable change of the semicrystalline structure of a polymer material.

\section{Nanostructure Differences as Deduced from the CDF}

Figure 2 presents a pseudocolor representation of the absolute value of the multidimensional chord 


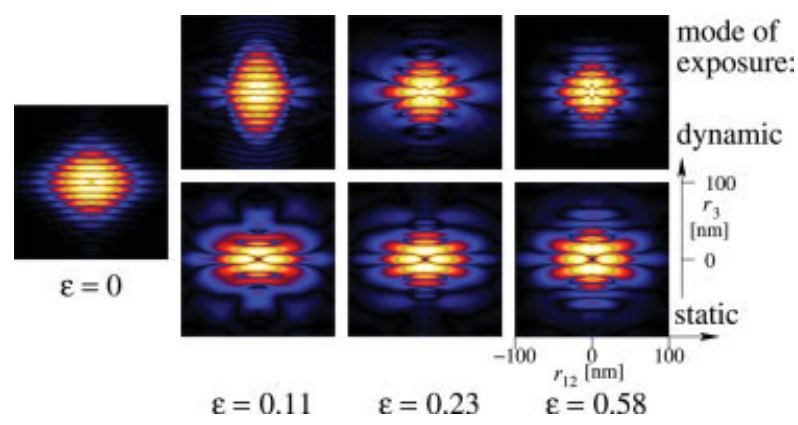

Figure 2. Nanostructure differences in hard-elastic PP films subjected to a true dynamic tensile test (top row) and a tensile test in stretch-hold technique (bottom row). The patterns show the absolute value of the CDF on a logarithmic scale, $\log \left|z\left(r_{12}, r_{3}\right)\right| . r_{3}$ is fiber axis and straining direction. $r_{12}$ is equatorial direction.

distribution function, $z\left(r_{12}, r_{3}\right)$. In the unstrained state $(\varepsilon=0)$ many parallel peaks of triangular shape are observed. This feature is indicating a stack of lamellae, because the CDF signal at $\mathbf{r}=\left(r_{12}, r_{3}\right)$ is approximately proportional to the area of surface contact between the domain structure and its ghost displaced by $\mathbf{r}$. The triangular decrease of CDF-intensity in the equatorial $r_{12}$ direction is readily established from the almost linear decrease of the area of contact, as the ghost surface is sliding off the real surface in analogy to the area of contact between your left and right palm of hand, as one of it is sliding past the other. The series of observed peaks is a consequence of the stacking - with increasing $r_{3}$ the ghost is probing layer surfaces, again and again.

In the dynamic experiment at $\varepsilon=0.11$, the length of the lines has considerably decreased, showing disruption of the lamellae. Nevertheless, the longitudinal correlation among the lamellae is still strong. At higher elongations the longitudinal correlation among the lamellae is shorter, and the layer thickness distributions of the lamellae grow somewhat broader.

Switching to the bottom row shows that, at all elongations, the width of the layer thickness distributions is even wider if the scattering is measured after stopping the extensometer. Moreover, the lateral extension of the lamellae is wider when measured at constant elongation (cf. Fig. 3). Thus, we conclude that the disruption of lamellae (i.e. a loss of average lateral extension) is partly compensated as soon as the extensometer is stopped. During drawing the population of crystallites is rather uniform. After stopping the machine some of the crystallites grow thick while others remain thin and the correlation among the lamellae becomes restricted to their next neighbors.

Finally, let us identify the nanostructural feature that is related to the different strength of the equatorial streak in the scattering patterns. The corresponding domains are easily identified in surface plots of the CDFs (cf. Fig. 3). Comparing the plots one observes that the strength of the equatorial streak is proportional to the strength of bridge peaks running parallel to the meridian, $r_{3}$, and connecting the lamellae peaks. These bridges are weaker or even not present in the data from the dynamic experiment. They are readily explained by cross-hatched lamellae, which are a common feature of PP. As has been pointed out by one of the manuscript reviewers, the bridge formation is decreasing with increasing strain for the static experiments. This finding may indicate that the ability to bridge the amorphous gap is a function of the gap between the primary crystalline lamellae.

Whenever bridge-formation is observed in the $\mathrm{CDF}$, the equatorial streak does no longer exhibit a continuous decay along the meridian, but wellresolved maxima. This is readily explained by the correlation among the crystalline bridges in equatorial direction. ${ }^{43}$ These observations support a finding from a previous study ${ }^{43}$ which indicated that the cross-hatched lamellae of PP are a secondary feature of its crystallization. We admit that this observation should be verified by a corresponding WAXS study.

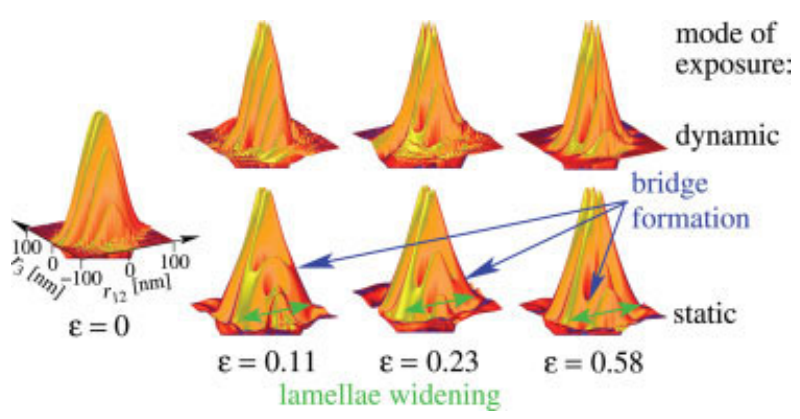

Figure 3. Nanostructure differences in hard-elastic PP films subjected to a true dynamic tensile test (top row) and a tensile test in stretch-hold technique (bottom row). The surface plots show the $\mathrm{CDF} z\left(r_{12}, r_{3}\right)$ on a logarithmic scale. $r_{3}$ is fiber axis and straining direction. $r_{12}$ is equatorial direction.

Journal of Polymer Science: Part B: Polymer Physics DOI $10.1002 /$ polb 


\section{CONCLUSIONS}

Only since it has become possible to record low-noise SAXS patterns within short time, an in-depth discussion of nanostructure evolution in polymer materials during dynamic mechanical tests has come into reach. As indicated by the presented results, the combination with the CDF data analysis is becoming a powerful method of nanostructure analysis.

We have demonstrated that the results obtained by means of the former stretch-hold technique may yield completely different results. Thus, without verification in a dynamic experiment they can hardly help to understand or to model the practical dynamic structure evolution of the material. On the other hand, if the monitoring by $\mathrm{X}$-ray scattering is combined both with dynamic mechanical testing and stretch-hold experiments, one can obtain important additional information concerning the nanostructure relaxation mechanisms that take place immediately after stopping the extensometer.

The observed big structure relaxation may be a result of a considerable decrease of stress after stopping the extensometer, which is typical for the semicrystalline hard-elastic materials. Thus, the demonstrated differences between stretchhold experiments and dynamic experiments may be larger than with typical elastomers which show less stress relaxation. Unfortunately, we have not monitored the stress relaxation in our stretch-hold experiments. The monitored stress data from our dynamic experiments will be part of a separate publication.

Presently, only dynamic mechanical tests with low strain rate can be combined with low-noise SAXS monitoring, and the strain rates applied in industrial applications are higher by factors of 10-100. Nevertheless, the technological gap is narrowing. By the time when the number of 3rd generation synchrotron sources has grown and the newly advertised high-power detectors will be available for materials research, the cycle time should decrease by a factor of 60 to $1 \mathrm{~s}$. Reciprocally, the maximum $\dot{\varepsilon}$ will increase.

We thank the Hamburg Synchrotron Radiation Laboratory (HASYLAB) for beam time granted in the frame of project II-01-041. We gratefully acknowledge funding by the Deutsche Forschungsgemeinschaft, project STR 501/4-2.

\section{REFERENCES AND NOTES}

1. Wu, J.; Schultz, J. M.; Yeh, F.; Hsiao, B. S.; Chu, B. Macromolecules 2000, 33, 1765-1777.

2. Hughes, D. J.; Mahendrasingam, A.; Martin, C.; Oatway, W. B.; Heeley, E. L.; Bingham, S. J.; Fuller, W. Rev Sci Instrum 1999, 70, 4051-4054.

3. Bras, W.; Mant, G. R.; Derbyshire, G. E.; O’Kane, W. J.; Helsby, W. I.; Hall, C. J.; Ryan, A. J. J Synchrotron Radiat 1995, 2, 87-92.

4. Butler, M. F.; Donald, A. M.; Bras, W.; Mant, G. R.; Derbyshire, G. E.; Ryan, A. J. Macromolecules 1995, 28, 6383-6393.

5. Butler, M. F.; Donald, A. M.; Ryan, A. J. Polymer 1997, 38, 5521-5538.

6. Hughes, D. J.; Mahendrasingam, A.; Oatway, W. B.; Heeley, E. L.; Martin, C.; Fuller, W. Polymer 1997, 38, 6427-6430.

7. Blundell, D. J.; Mahendrasingam, A.; McKerron, D.; Turner, A.; Rule, R.; Oldman, R. J.; Fuller, W. Polymer 1994, 35, 3875-3882.

8. Chen, X.; Yoon, K.; Burger, C.; Sics, I.; Fang, D.; Hsiao, B. S.; Chu, B. Macromolecules 2005, 38, 3883-3893.

9. Brandt, M.; Ruland, W. Acta Polym 1996, 47, 498506.

10. Liu, L.-Z.; Hsiao, B. S.; Fu, B. X.; Ran, S.; Toki, S.; Chu, B.; Tsou, A. H.; Agarwal, P. K. Macromolecules 2003, 36, 1920-1929.

11. Wilke, W.; Bratrich, M. J Appl Crystallogr 1991, 24, 645-650.

12. Grubb, D.; Bala, V. Polym Mater Sci Eng 1999, 81, 357-358.

13. Fu, B. X.; Hsiao, B. S.; Pagola, S.; Stephens, P.; White, H.; Rafailovich, M.; Sokolov, J.; Mather, P. T.; Jeon, H. G.; Phillips, S.; Lichtenhan, J.; Schwab, J. Polymer 2000, 42, 599-611.

14. Yeh, F.; Hsiao, B. S.; Sauer, B. B.; Michel, S.; Siesler, H. W. Macromolecules 2003, 36, 1940-1954.

15. Stribeck, N.; Polizzi, S.; Bösecke, P.; Zachmann, H. G. Rev Roumaine Chem 1989, 34, 635-648.

16. Stribeck, N.; Bösecke, P.; Polizzi, S. Colloid Polym Sci 1989, 267, 687-701.

17. Stribeck, N. J Polym Sci Part B: Polym Phys 1999, 37, 975-981.

18. Stribeck, N.; Fakirov, S.; Sapoundjieva, D. Macromolecules 1999, 32, 3368-3378.

19. Fakirov, S.; Samokovlijsky, O.; Stribeck, N.; Apostolov, A. A.; Denchev, Z.; Sapoundjieva, D.; Evstatiev, M.; Meyer, A.; Stamm, M. Macromolecules 2001, 34, 3314-3317.

20. Stribeck, N.; Buzdugan, E.; Ghioca, P.; Serban, S.; Gehrke, R. Macromol Chem Phys 2002, 203, 636644.

21. Stribeck, N.; Androsch, R.; Funari, S. S. Macromol Chem Phys 2003, 204, 1202-1216. 
22. Stribeck, N.; Fakirov, S.; Apostolov, A. A.; Denchev, Z.; Gehrke, R. Macromol Chem Phys 2003, 204, 1000-1013.

23. Stribeck, N.; Funari, S. S. J Polym Sci Part B: Polym Phys 2003, 41, 1947-1954.

24. Sauer, B. B.; McLean, R. S.; Brill, D. J.; Londono, D. J. J Polym Sci Part B: Polym Phys 2002, 40, 1727-1740.

25. Murakami, S.; Yamakawa, M.; Tsuji, M.; Kohjiya, S. Polymer 1996, 37, 3945-3951.

26. Séguéla, R.; Prud'homme, J. Macromolecules 1988, 21, 635-643.

27. Lee, H. S.; Yoo, S. R.; Seo, S. W. J Polym Sci Part B: Polym Phys 1999, 37, 3233-3245.

28. Welsh, G. E.; Blundell, D. J.; Windle, A. H. J Mater Sci 2000, 35, 5225-5240.

29. Hernández, J. J.; García Gutiérrez, M. C.; Nogales, A.; Rueda, D. R.; Sanz, A.; Sics, I.; Hsiao, B. S.; Roslaniec, Z.; Broza, G.; Ezquerra, T. A. Polymer 2007, 48, 3286-3293.

30. Noether, H. D. Intern J Polymeric Mater 1979, 7, 57-82.

31. Sprague, B. S. J Macromol Sci Phys 1973, B8, 157-187.
32. Noether, H. D.; Whitney, W. Colloid Polym Sci 1973, 251, 991-1005.

33. Stribeck, N. J Appl Cryst 2001, 34, 496-503.

34. Stribeck, N.; Fakirov, S. Macromolecules 2001, 34, 7758-7761.

35. Stribeck, N. Fibre Text EE 2003, 11, 33-45.

36. Stribeck, N. In Condensation Thermoplastic Elastomers; Fakirov, S., Ed., Wiley-VCH: Weinheim, 2005; pp 197-225.

37. Stribeck, N. Anal Bioanal Chem 2003, 376, 608-617.

38. Stribeck, N.; Almendarez Camarillo, A.; Cunis, S.; Bayer, R. K.; Gehrke, R. Macromol Chem Phys 2004, 205, 1445-1454.

39. Stribeck, N. X-Ray Scattering of Soft Matter; Springer: Heidelberg, New York, 2007.

40. Stribeck, N.; Almendarez Camarillo, A.; Nöchel, U.; Schroer, C.; Kuhlmann, M.; Roth, S. V.; Gehrke, R.; Bayer, R. K. Macromol Chem Phys 2006, 207, 1239-1249.

41. Ruland, W. Colloid Polym Sci 1977, 255, 417-427.

42. Vonk, C. G. Colloid Polym Sci 1979, 257, 10211032.

43. Stribeck, N.; Nöchel, U.; Almendárez Camarillo, A.; Roth, S. V.; Dommach, M.; Bösecke, P. Macromolecules 2007, 40, 4535-4545. 